\title{
ON GERCHBERG'S METHOD FOR THE FOURIER INVERSE PROBLEM
}

\author{
T. J. CONNOLLY, K. A. LANDMAN and L. R. WHITE ${ }^{1}$
}

(Received 19 March 1992; revised 16 March 1994)

\begin{abstract}
If a finite segment of a spectrum is known, the determination of the finite object function in image space (or the full spectrum in frequency space) is a fundamental problem in image analysis. Gerchberg's method, which solves this problem, can be formulated as a fixed point iteration. This and other related algorithms are shown to be equivalent to a steepest descent method applied to the minimization of an appropriate functional for the Fourier Inversion Problem. Optimal steepest descent and conjugate gradient methods are derived. Numerical results from the application of these techniques are presented. The regularization of the problem and control of noise growth in the iteration are also discussed.
\end{abstract}

\section{Introduction}

Two long standing and fundamental problems in image restoration and reconstruction are the Fourier inversion problem and its natural partner the Fourier extrapolation problem (also known as super-resolution) [2, 28]. Both of these start with the assumption that only a finite segment of a Fourier transform is known, say $g(\omega)$ for $\omega \in[-\Omega, \Omega]$.

The Fourier Inversion Problem (FIP) requires the determination of the image function $f(x)$ such that

$$
\int_{-\infty}^{\infty} f(x) e^{-i 2 \pi \omega x} d x=g(\omega) \quad \text { for } \quad|\omega|<\Omega
$$

For reasons outlined below, Gerchberg [12] and Papoulis [26] studied this problem with the constraint that $f(x)$ is spatially limited (that is $f$ has compact support); that is

$$
f(x)=0 \quad \text { for } \quad|x|>X
$$

'Department of Mathematics, University of Melbourne, Parkville Victoria 3052, Australia.

(C) Australian Mathematical Society, 1995, Serial-fee code 0334-2700/95 
for some $X>0$.

The Fourier Extrapolation Problem (FEP) involves the recovery of the Fourier transform of $f(x)$ for frequencies outside the finite segment, hence for $|\omega|>\Omega$. This requires determining the transform $\hat{f}(\omega)$ for all $\omega$ such that in the finite segment

$$
\hat{f}(\omega)=g(\omega) \quad \text { for } \quad|\omega|<\Omega \text {. }
$$

It should be noted that in general, $g(\omega)$ will be made up of the Fourier transform of $f$ plus some additive noise $\epsilon(\omega)$ :

$$
g(\omega)=\hat{f}(\omega)+\epsilon(\omega), \quad|\omega|<\Omega .
$$

The two problems are strongly related. If the solution to the FIP is known then the FEP can be obtained by simply taking the Fourier transform

$$
\hat{f}(\omega)=\mathbf{F} f(x)=\int_{-\infty}^{\infty} f(x) e^{-i 2 \pi \omega x} d x, \quad|\omega|<\infty .
$$

Alternatively, if the solution to the FEP is known, then the image function can be reconstructed by

$$
f(x)=\mathbf{F}^{-1} \hat{f}(\omega)=\int_{-\infty}^{\infty} \hat{f}(\omega) e^{i 2 \pi \omega x} d \omega .
$$

Although we shall be chiefly concerned with solving the FIP, Gerchberg's method, by its very nature, produces solutions to both problems.

It is well-known that a compact support function has a Fourier transform which is an analytic function of $\omega$ and therefore uniquely determined outside $(-\Omega, \Omega)$. Hence the FEP and FIP have unique solutions. However analytic continuation is not a feasible method for the numerical solution of either problem.

In this paper we examine the use of Gerchberg's method [12] to solve the Fourier inversion and extrapolation problems. Gerchberg's method and its generalization ([2], page 74) proceeds by successively imposing the compact support constraint (and possibly extra constraints such as non-negativity) upon the solution in image space and then imposing the data in Fourier space. This iterative technique was also outlined by Papoulis [26]. Here we shall investigate the properties of this algorithm and its relationship to other iterative schemes such as Lent and Tuy [19] and gradient methods. The results of this analysis suggest improved algorithms for solution to these Fourier inversion and extrapolation problems. The ill-posed nature of the problem and control of the resulting noise growth in the iteration are also discussed. An extensive review of the application of iterative methods to the solution of inverse problems can be found in Connolly [7]. 


\section{Gerchberg's method as a fixed point iteration}

The Gerchberg algorithm [12] proceeds by successively imposing the measurement data in Fourier space and the compact support constraint in image space.

We take the initial estimate of the finite $f(x)$ in image space to be

$$
f^{(0)}(x)=0,
$$

or equivalently, the initial estimate of the spectrum $\hat{f}(\omega)$ in frequency space to be

$$
g^{(0)}(\omega)=g_{G}^{(0)}(\omega)= \begin{cases}g(\omega) & |\omega|<\Omega \\ 0 & |\omega|>\Omega\end{cases}
$$

Then the successive iterates of $f^{(k)}$ and $g^{(k)}$ for $k \geq 1$ can be constructed via

$$
g_{G}^{(k)}=g^{(0)}(\omega)+\mu(\omega) \mathbf{F} f_{G}^{(k)}(x)
$$

where

$$
\mu(\omega)= \begin{cases}0 & |\omega|<\Omega \\ 1 & |\omega|>\Omega\end{cases}
$$

and then the update for each compact support $f^{(k)}$ is

$$
f_{G}^{(k+1)}(x)=\gamma(x) \mathbf{F}^{-1} g_{G}^{(k)}(\omega),
$$

where

$$
\gamma(x)= \begin{cases}1 & |x|<X \\ 0 & |x|>X\end{cases}
$$

Therefore, starting with $f_{G}^{(k)}$ in image space, a new spectral update in frequency space is given by $g_{G}^{(k)}$ from (2.3), and then the next object function is $f_{G}^{(k+1)}$ from (2.5). However, for our purposes it is convenient to combine these as

$$
f_{G}^{(k+1)}=\gamma \mathbf{F}^{-1}\left(\mu \mathbf{F} f_{G}^{(k)}\right)+\gamma \mathbf{F}^{-1} g^{(0)} .
$$

This defines a fixed point iteration in image space between successive iterates $f_{G}^{(k)}$ and $f_{C}^{(k+1)}$. An alternative way of writing this, which will be referred to in Section 4 , shows explicitly the difference between successive iterates:

$$
f_{G}^{(k+1)}=f_{G}^{(k)}+\gamma \mathbf{F}^{-1}\left[g^{(0)}-\xi \mathbf{F} f_{G}^{(k)}\right]
$$

where

$$
\xi=(1-\mu)= \begin{cases}1 & |\omega|<\Omega \\ 0 & |\omega|>\Omega\end{cases}
$$


Similarly, a fixed point iteration for the spectrum in frequency space for the FEP is given by

$$
g_{G}^{(k+1)}=\mu \mathbf{F}\left(\gamma \mathbf{F}^{-1} g^{(k)}\right)+g^{(0)} .
$$

If additional information on the image function is known, for example upper and lower bounds on the function as

$$
l \leq f \leq u \quad|x|<X,
$$

then this constraint can be incorporated into a Gerchberg scheme via a projection $\mathbf{P}$ by

$$
\mathbf{P} f(x)= \begin{cases}l(x) & f<u \\ f(x) & l \leq f \leq u \\ u(x) & f>u\end{cases}
$$

for $|x|<X$. Clearly the case of a non-negativity constraint has $l=0$ and $u=\infty$. Then a generalized Gerchberg algorithm ([12], page 74) modifies (2.7) and (2.8) as

$$
\begin{aligned}
f_{G G}^{(k+1)} & =\mathbf{P}\left[\gamma \mathbf{F}^{-1}\left(\mu \mathbf{F} f_{G G}^{(k)}\right)+\gamma \mathbf{F}^{-1} g^{(0)}\right] \\
& =f_{G G}^{(k)}+\mathbf{P} \gamma \mathbf{F}^{-1}\left[g^{(0)}-\xi \mathbf{F} f_{G G}^{(k)}\right] .
\end{aligned}
$$

In the Appendix, it is shown that the mean square error in the solution is reduced with each iteration of the Gerchberg algorithm, that is,

$$
\left\|f-f^{(k+1)}\right\|_{L^{2}(-X, X)}<\left\|f-f^{(k)}\right\|_{L^{2}(-X, X)} .
$$

If a nonnegativity condition is imposed, this error reduction still holds.

\section{The Lent and Tuy method}

The Lent and Tuy algorithm [19] repeatly imposes each constraint on the image function $f$ by an associated projection operator. The essence of the scheme, in our notation, is set out below.

(a) The initial iterate is the backtransform of the finite spectral data in $|\omega|<\Omega$,

$$
f_{L T}^{(1)}=\mathbf{F}^{-1} g^{(0)}(\omega),
$$

where $g^{(0)}$ is defined in (2.2).

(b) Thereafter there are five steps in updating between successive iterates $f_{L T}^{(k)}$ and $f_{L T}^{(k+1)}$. Each step has an associated relaxation parameter $\lambda_{i}^{(k)}(i=1, \ldots, 5)$ which determines how strongly each constraint is imposed. The constraints and steps are as follows: 
(i) $f$ real:

$$
f_{1}^{(k)}=f_{L T}^{(k)}+\lambda_{1}^{(k)}\left[\operatorname{Re}\left(f_{L T}^{(k)}\right)-f_{L T}^{(k)}\right]
$$

(ii) $f$ has compact support:

$$
f_{2}^{(k)}=f_{1}^{(k)}+\lambda_{2}^{(k)}\left[\gamma f_{1}^{(k)}-f_{1}^{(k)}\right]
$$

(iii) $f$ has a lower bound $l(x)$ :

$$
f_{3}^{(k)}=f_{2}^{(k)}+\lambda_{3}^{(k)}\left[\sup \left(l, f_{2}^{(k)}\right)-f_{2}^{(k)}\right]
$$

(iv) $f$ has an upper bound $u(x)$ :

$$
f_{4}^{(k)}=f_{3}^{(k)}+\lambda_{4}^{(k)}\left[\inf \left(u, f_{3}^{(k)}\right)-f_{3}^{(k)}\right]
$$

(v) Given spectral data $g(\omega)$ in $|\omega|<\Omega$ :

$$
f_{L T}^{(k+1)}=\mathbf{F}^{-1}\left[\lambda_{5}^{(k)} g^{(0)}+\left(1-\lambda_{5}^{(k)}\right) \xi \mathbf{F} f_{4}^{(k)}+\mu \mathbf{F} f_{4}^{(k)}\right]
$$

The authors prove that if $0<\lambda_{i}^{(k)}<2$ for $i=1, \ldots, 5$, the iterates $f_{L T}^{(k)}$ and $\mathbf{F} f_{L T}^{(k)}$ converge weakly to solutions of the FIP and FEP respectively.

Note in particular that in this scheme, the Fourier data in the interval $|\omega|<\Omega$ is replaced by

$$
\lambda_{5}^{(k)} g+\left(1-\lambda_{5}^{(k)}\right) \mathbf{F} f_{4}^{(k)}
$$

(using (v) above). If for a particular constraint $\lambda_{i}^{(k)}$ satisfies $0<\lambda_{i}^{(k)}<1$, then that constraint is under-relaxed at the $k$ th iteration, while if $\lambda_{i}^{(k)}$ satisfies $1<\lambda_{i}^{(k)}<2$ then the constraint is over-relaxed. The special case when all five relaxation parameters are unrelaxed, that is $\lambda_{i}^{(k)} \equiv 1$, makes the algorithm equivalent to the generalized Gerchberg method discussed in Section 2, with $f_{4}^{(k)}=\mathbf{P} \gamma f_{L T}^{(k)}=f_{G G}^{(k)}$. If $l=-\infty$ and $u=\infty$, steps (iii) and (iv) in the above procedure collapse, and again choosing the remaining relaxation parameters to be unity, then the Lent and Tuy algorithm reduces to the original Gerchberg algorithm with $f_{2}^{(k)}=\gamma f_{L T}^{(k)}=f_{G}^{(k)}$.

Lent and Tuy utilize the algorithm to numerically solve a two-dimensional limitedangle $X$-ray tomography problem; however the values of the relaxation parameters are not specified. The authors note an enhancement of the quality of the image obtained with the incorporation of available $a$ priori knowledge via the above constraints. Davies et al. [8] utilized the unrelaxed Lent and Tuy method (that is $\lambda_{i}^{(k)} \equiv 1$ ) to solve Fourier convolution integral equations with a non-negativity constraint on the solution.

The Lent and Tuy method is based upon a projection method suggested by Bregman [6] which is known to converge. Vasin [30] discusses a general method for 
including convex constraints with an iterative method to solve an ill-posed problem, and includes conditions for convergence.

For the case when only the compact support constraint is imposed, it is convenient to explicitly show the difference between successive iterates as

$$
f_{L T}^{(k+1)}=\gamma f_{L T}^{(k)}+\lambda^{(k)} \mathbf{F}^{-1}\left[g^{(0)}-\xi \mathbf{F} \gamma f_{L T}^{(k)}\right]
$$

using (3.6) with $\lambda^{(k)}=\lambda_{5}^{(k)}$ and $f_{4}^{(k)}=\gamma f_{L T}^{(k)}$.

In this form it is clear that Gerchberg's method (and more generally the Lent and Tuy method with compact support constraint only) is an example of Landweber's iteration [18] solving first kind integral equations. Convergence then follows for all $0<\lambda \leq 2$ as $\|F\| \leq 1$, as in Schock [27].

\section{Relationship with a steepest descent method}

In this section, the Fourier inverse problem is formulated as a minimization problem in $L^{2}(-X, X)$ and a steepest descent method for the problem is derived. This highlights the close relationship between a steepest descent method and Gerchberg's method and furthermore points to ways of improving Gerchberg's method.

The steepest descent method minimizes a functional $P(f)$ using an iterative scheme ([21], page 149)

$$
\begin{aligned}
f_{S D}^{(k+1)} & =f_{S D}^{(k)}+\alpha^{(k)} s^{(k)}, \\
s^{(k)} & =-\nabla P\left(f_{S D}^{(k)}\right),
\end{aligned}
$$

where $s^{(k)}$ is the steepest descent direction, $\nabla P\left(f_{S D}^{(k)}\right)$ is the gradient of $P$ with respect to $f$ and $\alpha^{(k)}$ is the steplength (which may be determined by linear search or other means).

For the FIP, (1.1) and (1.2) suggest an appropriate functional to be minimized as

$$
P(f)=\int_{-\Omega}^{\Omega}\left|\int_{-X}^{X} f(x) e^{-i 2 \pi \omega x} d x-g(\omega)\right|^{2} d \omega
$$

for functions $f \in L^{2}(-X, X)$. Suitable constraints on $f$, for example upper and lower bounds as in (2.11), may be imposed.

It is convenient to introduce the following notation: let $A$ be the operator acting on functions in $L^{2}(-X, X)$ such that

$$
A f=\int_{-X}^{X} f(x) e^{-i 2 \pi \omega x} d x \quad \text { for } \quad|\omega|<\Omega
$$


(Clearly if $A$ is defined on $L^{2}(-\infty, \infty)$ for functions with compact support satisfying (1.2), then $A=\mathbf{F})$. Then the functional $P(f)$ in (4.3) can be written as

$$
P(f)=\|A f-g\|_{L^{2}(-\Omega, \Omega)}=\langle A f-g, A f-g\rangle_{L^{2}(-\Omega, \Omega)} .
$$

On expanding the inner product, it is clear that

$$
\min _{f \in L^{2}(-X, X)} P(f) \equiv \min _{f \in L^{2}(-X, X)}\left\langle f, \frac{1}{2} Q f-b\right\rangle,
$$

where

$$
Q=2 A^{*} A, \quad b=2 A^{*} g
$$

and $A^{*}$ is the adjoint operator of $A$, acting on $L^{2}(-\Omega, \Omega)$

$$
A^{*} g=\int_{-\Omega}^{\Omega} g(\omega) e^{i 2 \pi \omega x} d \omega \quad \text { for } \quad|x|<X .
$$

Hence, (4.6) is in the usual form for the minimization of a quadratic functional. Standard theory ([21], page 149) gives the steepest descent direction $s^{(k)}$ at iteration $k$ as

$$
s^{(k)}=-\left(Q f_{S D}^{(k)}-b\right)=2 A^{*} g-2 A^{*} A f_{S D}^{(k)} .
$$

By extending the definition of $f_{S D}^{(k)}$ and $s^{(k)}$ from $L^{2}(-X, X)$ to $L^{2}(-\infty, \infty)$ by requiring the function to be zero outside $|x|>X,(4.9)$ can be written as

$$
s^{(k)}=2 \gamma \mathbf{F}^{-1}\left[g^{(0)}-\xi \mathbf{F} f_{S D}^{(k)}\right] .
$$

Hence (4.1) becomes

$$
f_{S D}^{(k+1)}=f_{S D}^{(k)}+2 \alpha^{(k)} \gamma \mathbf{F}^{-1}\left[g^{(0)}-\xi \mathbf{F} f_{S D}^{(k)}\right] .
$$

If upper and lower bounds on $f((2.11))$ are implemented by projection $\mathbf{P}((2.12))$, then a projected steepest descent method can be applied as

$$
f_{P S D}^{(k+1)}=f_{P S D}^{(k)}+2 \alpha^{(k)} \mathbf{P} \gamma \mathbf{F}^{-1}\left[g^{(0)}-\xi \mathbf{F} f_{P S D}^{(k)}\right] .
$$

Now some interesting comparisons can be made. Upon setting $\alpha^{(k)}=0.5,(4.11)$ and (4.12) are just (2.8) and (2.13b) respectively. Hence Gerchberg's (generalized Gerchberg's) method is equivalent to a steepest descent (projected steepest descent) method with fixed steplength $\alpha^{(k)}=0.5$. Comparison between the Lent and Tuy and steepest descent algorithms, using (3.8) and (4.11) can be made by identifying the relaxation parameter $\lambda^{(k)}$ with the steplength as

$$
\lambda^{(k)}=2 \alpha^{(k)} .
$$


TABLE 1. Equivalence of iterative methods

\begin{tabular}{|c|c|c|c|c|}
\hline Steepest Descent & & Gerchberg/Papoulis & & Lent and Tuy \\
\hline $\begin{array}{c}\text { Step Length } \\
\alpha^{(k)}=0.5\end{array}$ & $\Leftrightarrow$ & Gerchberg & $\Leftrightarrow$ & $\begin{array}{c}\text { Constraint (ii) and (v) only } \\
\lambda_{2}^{(k)}=\lambda_{5}^{(k)}=1\end{array}$ \\
\hline $\begin{array}{c}\text { Projected } \\
\text { Step Length } \\
\alpha^{(k)}=0.5\end{array}$ & $\Leftrightarrow$ & $\begin{array}{c}\text { Generalized } \\
\text { Gerchberg }\end{array}$ & $\Leftrightarrow$ & $\lambda_{i}^{(k)}=1, i=1, \ldots, 5$ \\
\hline $\begin{array}{l}\text { Step Length } \\
0<\alpha^{(k)}<1\end{array}$ & & $\Leftrightarrow$ & & $\begin{array}{c}\text { Constraint (ii) and (v) only } \\
\lambda_{2}^{(k)}=1 \\
\lambda_{5}^{(k)}=2 \alpha^{(k)}\end{array}$ \\
\hline $\begin{array}{c}\text { Projected } \\
\text { Step Length } \\
0<\alpha^{(k)}<1\end{array}$ & & $\Leftrightarrow$ & & $\begin{array}{c}\lambda_{i}^{(k)}=1, i=1, \ldots, 4 \\
\lambda_{5}^{(k)}=2 \alpha^{(k)}\end{array}$ \\
\hline
\end{tabular}

Hence, when only the compact support constraint is imposed, the Lent and Tuy scheme is equivalent to a steepest descent method with a choice for each iteration number $k$ of a steplength $\alpha^{(k)}$ such that $0<\alpha^{(k)}<1$ for convergence. In Table 1 we summarize the conclusions made here and in the previous section.

The fact that Gerchberg's method is formally equivalent to a steepest descent method for minimizing our functional $P(f)$ explains its relatively slow rate of asymptotic convergence. Gerchberg [12] states that the error "characteristically converges rapidly toward zero at first and more slowly as the limit is approached".

We note that for the more general Landweber iteration, convergence may also be arbitrarily slow [27], with the convergence rate dependent upon the smoothness of the data $g$.

\section{Optimal steepest descent and conjugate gradients}

As discussed above, the various Gerchberg and Lent and Tuy algorithms can be expressed as steepest descent methods. We wish to exploit this relationship by obtaining more efficient algorithms for solving the FIP and FEP.

One direction to follow is to find the optimal steplength $\alpha^{(k)}$. A standard result 
([21], page 150$)$ for minimizing $\left(f, \frac{1}{2} Q f-b\right)$ gives this as

$$
\alpha^{(k)}=\frac{\left\langle s^{(k)}, s^{(k)}\right\rangle_{L^{2}(-X, X)}}{\left\langle s^{(k)}, Q s^{(k)}\right\rangle_{L^{2}(-X, X)}}=\frac{\left\|s^{(k)}\right\|_{L^{2}(-X, X)}^{2}}{2\left\|A s^{(k)}\right\|_{L^{2}(-\Omega, \Omega)}^{2}}
$$

using (4.7) for $Q$. Hence for a known direction $s^{(k)}, \alpha^{(k)}$ can be easily calculated. If $\Omega=\infty, A=\mathbf{F}$ (the Fourier transform operator), and hence Parseval's identity gives $\alpha^{(k)} \equiv 0.5$ for each $k$. (This is the value for the Gerchberg algorithm.) More generally, for $\Omega<\infty$, since

$$
\left\|A s^{(k)}\right\|_{L^{2}(-\Omega, \Omega)}^{2}<\left\|\mathbf{F} s^{(k)}\right\|_{L^{2}(-\infty, \infty)}^{2}=\left\|s^{(k)}\right\|_{L^{2}(-\infty, \infty)}^{2}
$$

therefore

$$
\alpha^{(k)}>0.5 \quad \text { for } \quad \Omega<\infty .
$$

Hence in terms of the Lent and Tuy algorithm, at each iteration the optimal steepest descent direction corresponds to an over-relaxation of the constraint specifying the given Fourier data in $|\omega|<\Omega$.

We see from (4.11) and (5.1) that the Fourier transforms $\mathbf{F} f_{S D}^{(k)}$, $\mathbf{F}^{-1}\left(g^{(0)}-\xi \mathbf{F} f_{S D}^{(k)}\right)$ and $\mathbf{F} s^{(k)}$ are required for the calculation of the update $f_{S D}^{(k+1)}$ at each iteration. However since

$$
f_{S D}^{(k)}=f_{S D}^{(k-1)}+\alpha^{(k-1)} s^{(k-1)}
$$

the $\mathbf{F} f_{S D}^{(k)}$ can be computed from the already determined $\mathbf{F} f_{S D}^{(k-1)}$ and $\mathbf{F} s^{(k-1)}$ at the previous iteration. Thus $\mathbf{F} f_{S D}^{(k)}$ does not need to be computed by fast Fourier transforms and the computation of only two Fourier transforms per iteration is required as with Gerchberg's method.

The other way to improve the asymptotic rate of convergence of the steepest descent method is by trying a conjugate gradient method ([21], page 174). The successive iterations in this scheme are obtained by

$$
f_{C G}^{(k+1)}=f_{C G}^{(k)}+\delta^{(k)} t^{(k)}
$$

where the conjugate gradient descent direction must be determined by

$$
t^{(k+1)}=s^{(k+1)}+\beta^{(k)} t^{(k)}
$$

from the steepest descent direction $s^{(k)}$ given by

$$
s^{(k)}=b-Q f_{C G}^{(k)}=2 \gamma \mathbf{F}^{-1}\left[g^{(0)}-\xi \mathbf{F} f_{C G}^{(k)}\right],
$$

with a choice

$$
t^{(0)}=s^{(0)}=b-Q f^{(0)}=2 \gamma \mathbf{F}^{-1} g^{(0)}
$$


So the initial step is identical to the steepest descent step, but each succeeding step is a linear combination of the current gradient and the preceding direction vector. The steplengths $\delta^{(k)}$ and $\beta^{(k)}$ are determined as

$$
\begin{gathered}
\delta^{(k)}=\frac{\left\langle s^{(k)},\left.s^{(k)}\right|_{L^{2}(-X, X)}\right.}{\left\langle t^{(k)}, Q t^{(k)}\right\rangle_{L^{2}(-X, X)}}=\frac{\left\|s^{(k)}\right\|_{L^{2}(-X, X)}^{2}}{2\left\|A t^{(k)}\right\|_{L^{2}(-\Omega, \Omega)}^{2}}, \\
\beta^{(k)}=\frac{\left\|s^{(k+1)}\right\|^{2}}{\left\|s^{(k)}\right\|^{2}} .
\end{gathered}
$$

As with the optimal steepest descent method, only two Fourier transforms per iteration are required for this conjugate gradient method. Error estimates for the convergence of both the steepest descent and conjugate gradient methods for the least squares problem for $A f=g$ are given in Kammerer and Nashed [17, 18], and later improved for conjugate gradients in Louis[20] and Brakhage [5] with smoother data.

In the Fourier phase problem, when the phase of a Fourier transform is to be determined from knowledge of the amplitude of the transform, Fienup's method [10] successively iterates between image and Fourier spaces, imposing constraints in each space, just like Gerchberg's method for FIP. Fienup [11] shows that the steepest descent method for an appropriate functional for the Fourier phase space problem is equivalent to a variation of Fienup's method known as the "error reduction method". He states that this method has fast convergence initially but slow asymptotically. Conjugate gradient methods are suggested but not implemented to increase this rate of convergence.

The results of numerical calculations using these various iteration methods are discussed in Section 6, allowing comparisons to be made. In the previous sections we have only discussed the Fourier inversion problem. Essentially the same analysis follows for reconstructing the Fourier transform $\hat{f}(\omega)=\lim _{k \rightarrow \infty} g^{(k)}(\omega)$.

\section{Numerical results}

The theoretical differences between the various algorithms discussed above can be demonstrated by considering a few types of image functions $f(x)$. We choose

(i) a Gaussian - no compact support, smooth function;

(ii) a triangular function - compact support, discontinuities in derivative;

(iii) a rectangular function - compact support, discontinuities.

Clearly, depending on the application, different properties of $f(x)$ will be appropriate. For example, in the gas absorption problems considered by Lum Wan and White $[23,22]$, the functions are probability functions and therefore of Gaussian type. 
Various quantities can be used as measures of the quality of the algorithm. These are the mean error in $f(x)$ for FIP and the mean error in $\hat{f}(\omega)$ for FEP at the $k$ th iteration:

$$
\left[\int_{-X}^{X}\left|f-f^{(k)}\right|^{2} d x\right]^{1 / 2} \quad \text { and } \quad\left[\int_{-\infty}^{\infty}\left|\hat{f}-g^{(k)}\right|^{2} d \omega\right]^{1 / 2}
$$

respectively. The integrals are replaced by the appropriate sums for the discrete numerical calculations. Also of interest are the mean energy of the $k$ th iterates

$$
\left[\int_{-X}^{X}\left|f^{(k)}\right|^{2} d x\right]^{1 / 2} \quad \text { and } \quad\left[\int_{-\infty}^{\infty}\left|g^{(k)}\right|^{2} d \omega\right]^{1 / 2}
$$

and the peak height of each $f^{(k)}$.

First, let us consider the case when the image function is taken to be Gaussian. Therefore the given Fourier data comes from $\hat{f}(\omega)=e^{-(\pi \omega / A)^{2}} e^{-2 \pi i \omega B}$ for the image function

$$
f(x)=\sqrt{\frac{A}{\pi}} e^{-A(x-B)^{2}}
$$

Several parameters can be varied, namely $A, B$, the frequency cutoff $\Omega$ and the compact support dimension $X$. (In all examples, the spacings $\delta \omega$ and $\delta x$ for the discrete values of $f^{(k)}(x)$ and $g^{(k)}(\omega)$ are chosen so that the functions are well defined, that is well resolved. This can be verified by varying $\delta \omega$ and $\delta x-$ the results will be insensitive to small changes in $\delta \omega$ and $\delta x$ if they are chosen correctly). Noise free and noisy data $g^{(0)}(\omega)$ are considered separately.

(1) Examples with no noise

In our first example $A=1, B=0$ and the cutoff frequency value is $\Omega=0.3183$ giving $\hat{f}(\Omega)=\hat{f}(0) e^{-1}$. In Figures $1 \mathrm{a}$ and $1 \mathrm{~b}$, the mean error is $f(x)$ and $\hat{f}(\omega)$ at the $k$ th iterate are plotted using the Gerchberg, Lent and Tuy $\left(\lambda^{(k)}=2 \alpha^{(k)}=1.5\right)$, optimal steepest descent and conjugate gradient algorithms. Each algorithm makes a large reduction in error for the first few iterations. The Gerchberg algorithm has the slowest error reduction. As anticipated by our theoretical discussions the error reduction is improved with the over-relaxed Lent and Tuy algorithm, again with optimal steepest descent and finally the best reduction is with the conjugate gradient method. A perhaps surprising result is that the conjugate gradient method 'converges' in approximately eight iterations. This method takes 'discontinuous steps' in the mean error, whereas the other three methods exhibit an almost linear decline in mean error with iteration number. In this example the compact support constraint on the Gaussian was imposed for values of $|x|>3$, in that range $|f(x)|<1.2 \times 10^{-4}$. Of course, the compact support range of $f(x)$ is often unknown in a real example, or the function may not have compact support (as for Gaussian) but only tend to zero as $|x| \rightarrow \infty$. Clearly, the 


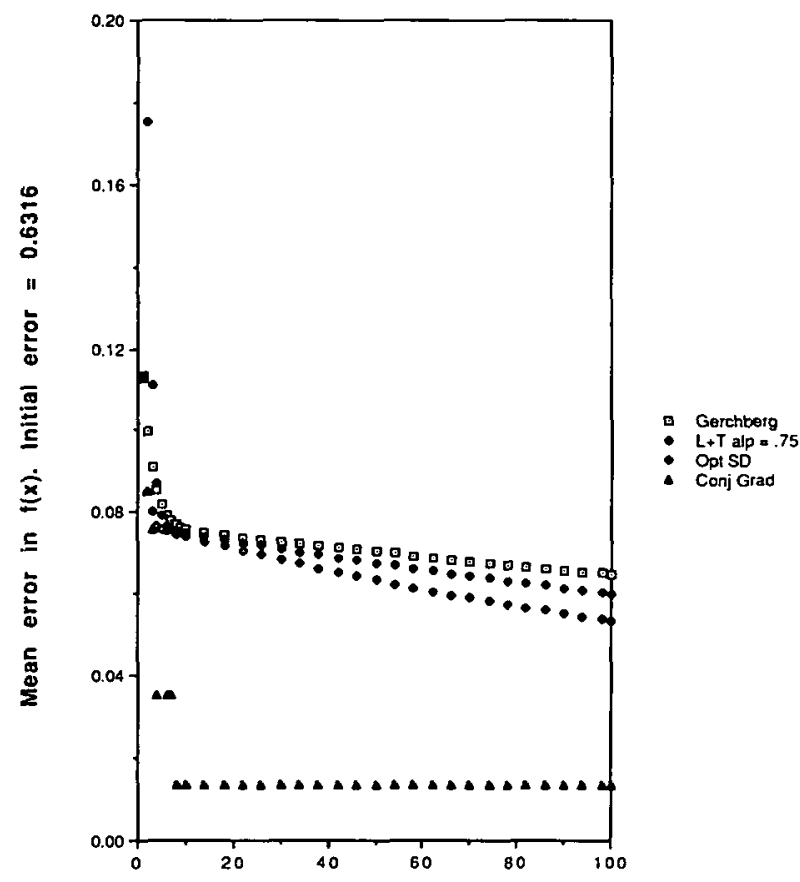

Iteration number

FIGURE 1a: Mean error in Gaussian $f(x)=\pi^{-1 / 2} e^{-x^{2}} . X=3, \Omega=0.318$ using various iterative techniques. No noise.

results of the FIP must be consistent for various values of support length $X$. However, if the support length is chosen too small, it would be hoped that the results become inconsistent in some way. For example, when the support length $X$ was halved to 1.5 in the above example, the error in the conjugate gradient method decreases for the first three iterations but then increases to a value larger than the initial value. (This behaviour also occurs in the other methods but for smaller values of $X$.)

However, it is clear that the mean error is not in practice an appropriate measure of the accuracy of the method because the exact $f(x)$ and $\hat{f}(\omega)$ are unknown in a real problem. Other measures considered were the supposed peak height, $f^{(k)}(0)$ here, and the mean energy. When the compact support length was chosen large enough, $f^{(k)}(0)$ increased monotonically with $k$. However when $X=1.5, f^{(k)}(0)$ initially increased but then decreased rapidly at large values of $k$. Indeed in this case the origin was not the position of a maximum for $f^{(k)}(x)$. Hence both the nature of $f^{(k)}(x)$ and its value at the origin become inconsistent if the compact support length is too small. (The same behaviour is seen in the other methods for smaller values of $X$.) 


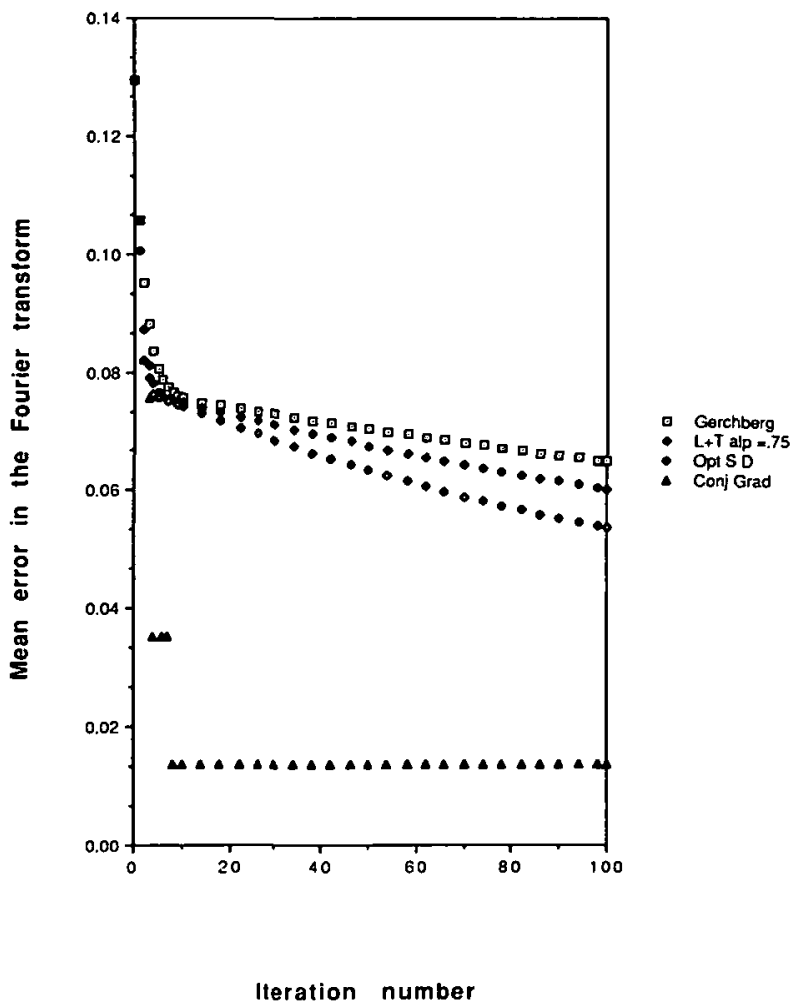

FIGURE 1b: Mean error in Fourier transform $\hat{f}(w)=e^{-\pi^{2} \omega^{2}} . X=3, \Omega=0.318$ and various iterative techniques. No noise.

We also considered having less initial Fourier data by decreasing the frequency cutoffs (for example $\Omega=0.212, \hat{f}(\Omega)=0.641$ ). All the results were qualitatively the same as those above, although as expected the mean error at each iteration $k$ is larger for the smaller cutoff for each of the methods.

In all the discussion above, the compact support region was taken to be $(-X, X)$. In fact there is no change in the analysis if the region is taken as $(a, b)$. This highlights the fact that both the length $(b-a)$ and range of the compact support is unknown. In the above example, we varied this region and the inconsistencies already discussed for too small values of $X$ also appear if the range is inappropriate. This naturally leads to the case of translated Gaussian, $(B \neq 0)$. In fact, this problem is no different from the centered Gaussian, despite oscillation in the Fourier transform, and qualitatively and quantitatively all the results hold. For example, as one would expect taking $A=B=1$ and range $(-2,4)$ with the same number of Fourier data points, the results are the same as for Figure la and $1 \mathrm{~b}$. More generally, cutoffs nonsymmetric about the peak in the function produce negligible difference to a symmetric cutoff 
provided the compact support approximation is valid.

For all the triangular and rectangular function examples, all the comments and results for the Gaussian function are true. Again the conjugate gradient method converges much more rapidly (approximately ten iterations) to mean errors considerably smaller (up to four times) the other methods (after 100 iterations). For both these examples, most of the error arises near the discontinuities in derivative (at $x=0$ for triangular function) and discontinuities in function value (at the ends for the rectangular function).

Positivity constraints were tried on these examples but did not improve the convergence rates. In addition, positivity often increased the mean error.

Of course the testing we have done is not all inclusive but it does indicate that the usual Gerchberg and Lent and Tuy methods can be improved by using optimal steepest descent or conjugate gradient methods.

(2) Examples with additive noise

The superiority of conjugate gradient methods for the solution of the FIP and FEP has been demonstrated when the initial Fourier data is noise free. In the above discussion, the only source of numerical error was roundoff and the use of the Fourier series approximation to the Fourier transform when using FFT's.

Gerchberg's method is well known not to converge in the presence of measurement noise in Fourier space. As the steps of the algorithm are linear, initial noise $\epsilon(\omega)$ has grown to

$$
\sum_{k=0}^{n}\left(\mu \mathbf{F} \gamma \mathbf{F}^{-1}\right)^{k} \epsilon
$$

after the $n$th iteration. Similar expressions for the propagation of noise can be obtained for the other iterative schemes.

We illustrate the growth in noise for the Gaussian example above and all parameters as in Figures 1a, 1b. The initial noise $\epsilon(\omega)$ added to $g^{(0)}(\omega)$ for $|\omega|<\Omega$ is the Fourier transform of pseudo-random numbers normally distributed with mean zero and variance $\sigma^{2}$. A standard deviation of $\sigma=\Sigma \%$ means that $\sigma=0.01 \Sigma f_{\max }$ where $f_{\max }$ is the largest value of $f(x)\left(=f(0)=\pi^{-1 / 2}\right.$ here $)$.

Introducing noise gives rise to competition between convergence to the solution and the growth of the noise. In each method the mean error curves initially decrease, reach a minimum and finally increase as the iteration number increases. Since the conjugate gradient method converges in approximately ten iterations in this example without noise, it is expected that the error amplification will occur at around this iteration number. The error amplification occurs later the slower the convergence rate, essentially due to the step length $\alpha^{(k)}$ being reduced. It is also notable that the mean error reduction is still smaller with conjugate gradient than the other methods. Some of these results are illustrated in Figure 2. The conjugate gradient method also has the advantage that by looking at the iterates $f^{(k)}$, it is clear between one or two 
successive iterates when the noise is swamping the solution. This is not the case with the other methods.

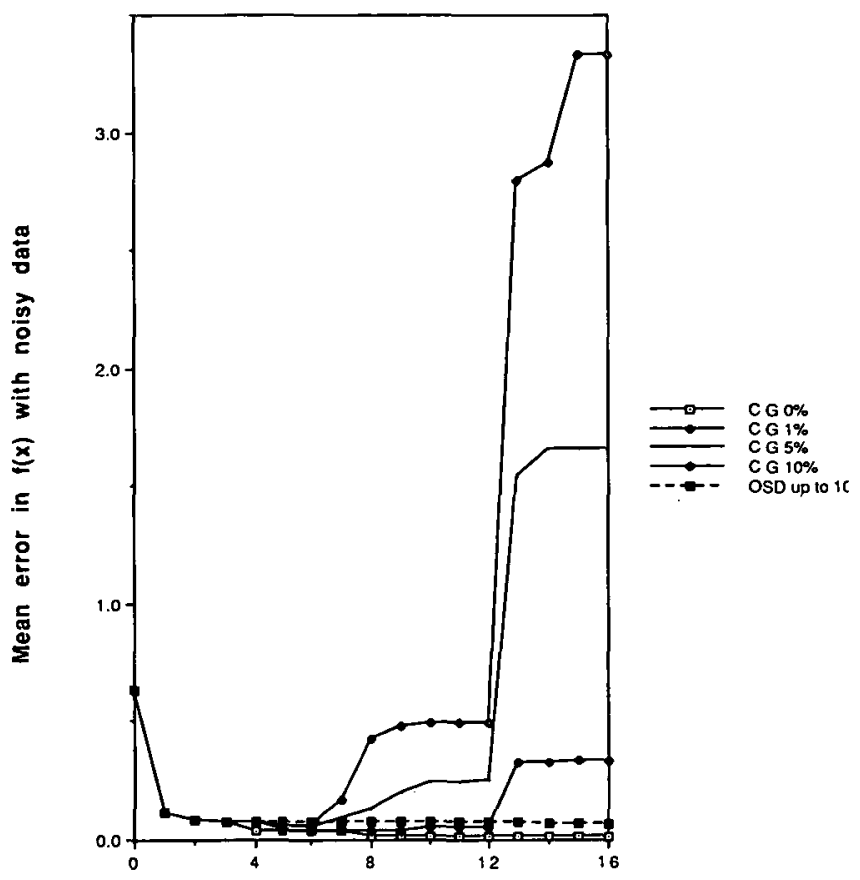

Iteration number

FIGURE 2. Mean error in Gaussian $f(x)=\pi^{-1 / 2} e^{-x^{2}} . X=3, \Omega=0.318$ with various noise levels using conjugate gradient and optimal steepest descent methods.

The sensitivity of the iterative methods to noise occurs because of the ill-posed nature of the inverse problems. Regularization methods could be used to stablize the methods and these are discussed further in Section 7.

\section{Discussion}

The existing Gerchberg and Lent and Tuy algorithm for the solution to the Fourier inverse and extrapolation problem have been rephrased as steepest descent methods. We have given numerical results illustrating the significantly improved rate of convergence and reduction in mean error of the solution obtained by generalising the iterative scheme to a conjugate gradient method. Although this method also suffers from noise amplification, it may not prove to be a major difficulty, as discussed in Section 6. 
We conclude by suggesting the following methods for the regularization and control of the noise growth.

(i) Limiting the number of iterations performed, with the number of iterations decreasing as the amount of noise increases. This stopping point may be chosen so that the iteration errors and amplified data errors balance $([14,13])$. In particular for the conjugate gradient method, stopping rules depending upon the residual $\left\|A f^{(k)}-g\right\|$ are given by Eicke $e t$ al. [9] and Nemirovskii [25].

(ii) Imposition of a constraint on the size of the solution, for example $|f(x)|<M$, using a projection, as in the Lent and Tuy method. It is a well-known result [3] that the problem is not ill-posed if the solution $f$ is in a known compact set; for the weak topology this also holds for the bounded set just suggested. An unbounded feasible set (such as a positivity constraint alone) may not help.

(iii) Tikhonov's regularization method [24]. This involves incorporating the constraint on $\|f\|$ into the functional being minimized in the descent method, for example,

$$
\|A f-g\|_{L^{2}(-\Omega, \Omega)}^{2}+\lambda\|f\|_{L^{2}(-X, X)}^{2},
$$

where the regularization parameter $\lambda$ depends on the amount of noise present. Methods for choosing this parameter are given by Miller [24] for example (and more recently [15]).

A stability result for the Fourier inverse problem regularized in this manner is to be found in [4]. To compute the minimum of the functional, the steepest descent or conjugate gradient methods (with a simple modification for the extra term in the functional) could be used. Convergence of these gradient methods would be guaranteed as the regularized functional has eigenvalues bounded away from zero.

\section{Appendix. Error reduction and non-negativity}

It was shown by Papoulis [26] that the mean-square error in the solution is reduced at each step of that iteration; that is

$$
\left\|f-\tilde{f}_{G}^{(k+1)}\right\|_{L^{2}(\mathbf{R})}<\left\|f-\tilde{f}_{G}^{(k)}\right\|_{L^{2}(\mathbf{R})},
$$

where $\tilde{f}_{G}^{(k)}$ denotes the object at each iteration before compact support is imposed:

$$
\tilde{f}_{G}^{(k+1)}=\mathbf{F}^{-1}\left(\mu \mathbf{F} f_{G}^{(k)}+g^{(0)}\right) .
$$

A complementary result can be obtained as

$$
\left\|f-f_{G}^{(k+1)}\right\|_{L^{2}(-X, X)}<\left\|f-f_{G}^{(k)}\right\|_{L^{2}(-X, X)}
$$


giving the mean-square error reduction for $f_{G}^{(k)}=\gamma \tilde{f}_{G}^{(k)}$, the object after compact support is imposed. This is proved by substituting $f=\mathbf{F}^{-1} \hat{f}$ and $g^{(0)}=\xi \hat{f}$ into equation (A1.1), using Parseval's identity and the compact support of $f, f_{G}^{(k)}, f_{G}^{(k+1)}$ :

$$
\begin{aligned}
\left\|f-f_{G}^{(k+1)}\right\|_{L^{2}(-X, X)} & <\left\|f-\tilde{f}_{G}^{(k+1)}\right\|_{L^{2}(\mathbf{R})} \\
& =\left\|\mu \hat{f}-\mu \mathbf{F} f_{G}^{(k)}\right\|_{L^{2}(\mathbf{R})} \\
& <\left\|\hat{f}-\mathbf{F} f_{G}^{(k)}\right\|_{L^{2}(\mathbf{R})} \\
& =\left\|f-f_{G}^{(k)}\right\|_{L^{2}(\mathbf{R})} \\
& =\left\|f-f_{G}^{(k)}\right\|_{L^{2}(-X, X)}
\end{aligned}
$$

We see from these steps that error reduction occurs twice, because of the two strict inequalities.

The effect upon the error reduction of imposing a non-negativity constraint on the solution $f$ is easily done. Let $\tilde{f}_{p}^{(k)}$ and $f_{p}^{(k)}$ be the $k$ th iterate solution before and after both the non-negativity and compact support constraints are imposed respectively. Then an argument similar to the one above gives one strict inequality in the relationship

$$
\left\|f-\tilde{f}_{p}^{(k+1)}\right\|_{L^{2}(\mathbf{R})}<\left\|f-f_{p}^{(k)}\right\|_{L^{2}(-X, X)},
$$

and the non-negativity of $f$ and $f_{p}^{(k+1)}$ gives

$$
\left\|f-f_{p}^{(k+1)}\right\|_{L^{2}(-X, X)} \leq\left\|f-\tilde{f}_{p}^{(k+1)}\right\|_{L^{2}(-X, X)}<\left\|f-\tilde{f}_{p}^{(k+1)}\right\|_{L^{2}(\mathbf{R})} .
$$

Combining these we obtain (with two and a possible three reductions per iteration)

$$
\left\|f-f_{p}^{(k+1)}\right\|_{L^{2}(-X, X)}<\left\|f-f_{p}^{(k)}\right\|_{L^{2}(-X, X)} \text {. }
$$

Error reduction will also follow (in a similar manner) for the imposition of known lower and upper bounds on the function.

Note that convergence of the iteration is not implied by these error reduction results. Convergence is proved by Papoulis [26] via the use of prolate spheriodal wave functions as basis functions. Also convergence properties can be gained by the relationship of Gerchberg's method to the Lent and Tuy and steepest descent methods and also Landweber's iteration. 


\section{References}

[1] R. A. Adams, Sobolev spaces (Academic, New York, 1975).

[2] R. H. T. Bates and M. J. McDonnell, Image restoration and reconstruction (Clarendon Press, Oxford, 1986).

[3] M. Bertero, "Regularization methods for linear inverse problems", Inverse problems. Lecture notes in mathematics 1225 (1986) 52-112.

[4] M. Bertero, C. De Mol and G. A. Viano, "The stability of inverse problems", in Inverse scattering problems in optics (ed. H. P. Baltes), (Springer Verlag, Berlin, 1979) 161-213.

[5] Helmut Brakhage, "On ill-posed problems and the method of conjugate gradients", in Inverse and ill-posed problems (eds Heinz W. Engl and C. W. Groetsch), (1987) 165-175.

[6] L. M. Bregman, "The method of successive projection for finding a common point in convex sets", Sov. Math. Dokl. 6 (1965) 688-692.

[7] T. J. Connolly, "Nonlinear methods for inverse problems", Ph. D. Thesis, University of Canterbury, Christchurch, New Zealand, 1990.

[8] A. R. Davies, M. Iqbal, K. Maleknejad and T. C. Redshaw, "A comparison of statistical regularization and Fourier extrapolation methods for numerical convolution", in Numerical treatment of inverse problems in differential and integral equations (eds P. Deufhlard and E. Hairer), (Birkhauser-Verlag, 1983) 320-334.

[9] B. Eicke, A. K. Louis and R. Plato, "The instability of some gradient methods for ill-posed problems", Numer. Math. 58 (1990) 129-134.

[10] J. R. Fienup, "Reconstruction of an object from the modulus of its Fourier transform", Optics Letters 3 (1978) 27-29.

[11] J. R. Fienup, "Phase retrieval algorithms: a comparison", Appl. Optics 21 (1982) 2758-2769.

[12] R. W. Gerchberg, "Super-resolution through error energy reduction", Optica Acta 21 (1974) 709720.

[13] F. A. Grünbaum, "A Study of Fourier space methods for limited angle image reconstruction", Numer. Funct. Anal. and Optimiz. 2 (1980) 31-42.

[14] M. Hanke, "Accelerated Landweber iterations for the solution of ill-posed problems", Numerische Matematik 60 (1991) 341-373.

[15] Per Christian Hansen, "Analysis of discrete ill-posed problems by means of the $L$-curve", SIAM Review 34 (1992) 561-580.

[16] W. J. Kammerer and M. Z. Nashed, "Steepest descent of singular linear operators with non-closed range", Applic. Anal. 1 (1971) 143-159.

[17] W. J. Kammerer and M. Z. Nashed, "On the convergence of the conjugate gradient method for singular linear operator equations", SIAM J. Numer. Anal. 9 (1972) 165-181.

[18] L. Landweber, "An iteration formula for Fredholm integral equations of the first kind", Amer. $J$. Math. 73 (1951) 615-624.

[19] A. Lent and H. Tuy, "An iterative method for the extrapolation of band-limited functions", J. of Math. Anal. and Applic. 83 (1981) 554-565.

[20] Alfred K. Louis, "Convergence of the conjugate gradient method for compact operators", in Inverse and ill-posed problems (eds Heinz W. Engl and C. W. Groetsch), (1987) 177-183.

[21] D. G. Luenberger, Introduction to linear and nonlinear programming (Addison-Wesley, Reading, 1965).

[22] J. A. Lum Wan and L. R. White, "A novel technique for the calculation of site energy density from gas absorption isotherm data", J. Chem. Soc. Faraday Trans. 87 (1991) 3051-3062.

[23] J. A. Lum Wan and L. R. White, "A numerical solution of inverse problems of Fourier convolution type", App. Math. Modelling 15 (1991) 359-366.

[24] K. Miller, "Least squares method for illposed problems with a prescibed bound", SIAM J. Math. 
Analy. 1 (1970) 52-74.

[25] A. S. Nemirovskii, "The regularizing properties of the adjoint gradient method in ill-posed problems", USSR Comput. Math. Math. Phys. 26 (2) (1986) 7-16.

[26] A. Papoulis, "A new algorithm in spectral analysis and band-limited extrapolation", IEEE Trans on Circuits and Systems CAS22 (1975) 735-742.

[27] E. Schock, "Comparison principles for iterative methods", in Inverse and ill-posed problems (eds Heinz W. Engl and C. W. Groetsch), (1987) 185-193.

[28] H. Stark (ed.), Image recovery: Theory and application (Academic Press, Orlando, 1981).

[29] A. N. Tikhonov and V. Y. Arsenin, Solutions of ill-posed problems (V. H. Winston, Washington D C, 1977).

[30] V. V. Vasin, "Iterative methods for the approximate solution of ill-posed problems with a priori information and their applications", in Inverse and ill-posed problems (eds Heinz W. Engl and C. W. Groetsch), (1987) 211-229.

[31] Grace Wahba, "Three topics in ill-posed problems", in Inverse and ill-posed problems (eds Heinz W. Engl and C. W. Groetsch), (1987) 37-51. 